\title{
Correction to: Acute lymphoblastic leukemia-derived exosome inhibits cytotoxicity of natural killer cells by TGF- $\beta$ signaling pathway
}

\author{
Huijun $\mathrm{Yu}^{1,2} \cdot$ Tingting Huang ${ }^{2} \cdot$ Daming Wang ${ }^{2} \cdot$ Lei Chen ${ }^{2} \cdot \mathrm{Xi} \mathrm{Lan}^{2} \cdot \mathrm{Xintong} \mathrm{Liu}^{2} \cdot \mathrm{Keyan} \mathrm{Chen}^{2} \cdot \mathrm{Haihong} \mathrm{He}^{2}$. \\ Shaobo $\mathrm{Li}^{2} \cdot$ Yiwen Zhou ${ }^{2}$. Jiansheng $\mathrm{Xie}^{1} \mathbb{C}$
}

Published online: 27 July 2021

(c) King Abdulaziz City for Science and Technology 2021

\section{Correction to: 3 Biotech (2021) 11:313 https://doi.org/10.1007/s13205-021-02817-5}

We apologize for the mistake of the affiliation 1. The corrected affiliation 1 should be "Shenzhen Maternity \& Child Healthcare Hospital, The First School of Clinical Medicine, Southern Medical University, 2004 Hongli Road, Futian District, Shenzhen, Guangdong, China".

The authors have found an error in funding information. The corrected funding information should be "This work was supported by Science, Technology and Innovation Bureau of Bao'an District of Shenzhen, Basic Research Project of Healthcare in Bao' an District of Shenzhen (No. 2019JD450)".
Funding This work was supported by Science, Technology and Innovation Bureau of Bao' an District of Shenzhen, Basic Research Project of Healthcare in Bao' an District of Shenzhen (No. 2019JD450).

The original article can be found online at https://doi.org/10.1007/ s13205-021-02817-5.

Jiansheng Xie

jsxieszmch@smu.edu.cn

1 Shenzhen Maternity \& Child Healthcare Hospital, The First School of Clinical Medicine, Southern Medical University, 2004 Hongli Road, Futian District, Shenzhen, Guangdong, China

2 Clinical Laboratory Medicine Center, Shenzhen Hospital, Southern Medical University, Shenzhen, Guangdong, China 